\title{
Design of passive coolers for light-emitting diode lamps using topology optimisation
}

\author{
Alexandersen, Joe; Sigmund, Ole; Meyer, Knud Erik; Lazarov, Boyan Stefanov
}

Published in:

International Journal of Heat and Mass Transfer

Link to article, DOI:

10.1016/j.jheatmasstransfer.2018.01.103

Publication date:

2018

Document Version

Early version, also known as pre-print

Link back to DTU Orbit

Citation (APA):

Alexandersen, J., Sigmund, O., Meyer, K. E., \& Lazarov, B. S. (2018). Design of passive coolers for lightemitting diode lamps using topology optimisation. International Journal of Heat and Mass Transfer, 122, 138149. https://doi.org/10.1016/j.ijheatmasstransfer.2018.01.103

\section{General rights}

Copyright and moral rights for the publications made accessible in the public portal are retained by the authors and/or other copyright owners and it is a condition of accessing publications that users recognise and abide by the legal requirements associated with these rights.

- Users may download and print one copy of any publication from the public portal for the purpose of private study or research.

- You may not further distribute the material or use it for any profit-making activity or commercial gain

- You may freely distribute the URL identifying the publication in the public portal 


\title{
Design of passive coolers for light-emitting diode lamps using topology optimisation
}

\author{
Joe Alexandersen ${ }^{\mathrm{a}, *}$, Ole Sigmund ${ }^{\mathrm{a}}$, Knud Erik Meyer ${ }^{\mathrm{b}}$, Boyan Stefanov Lazarov ${ }^{\mathrm{a}}$ \\ ${ }^{a}$ Department of Mechanical Engineering, Solid Mechanics \\ ${ }^{b}$ Department of Mechanical Engineering, Fluid Mechanics \\ Technical University of Denmark \\ Nils Koppels Allé, Building 404 \\ DK-2800, Denmark
}

\begin{abstract}
Topology optimised designs for passive cooling of light-emitting diode (LED) lamps are investigated through extensive numerical parameter studies. The designs are optimised for either horizontal or vertical orientations and are compared to a lattice-fin design. The different orientations result in significant differences in topologies. The optimisation favors placing material at outer boundaries of the design domain, leaving a hollow core that allows the buoyancy forces to accelerate the air to higher speeds. Investigations show that increasing design symmetry yields performance with less sensitivity to orientation with a minor loss in mean performance. The topology-optimised designs of heat sinks for natural convection yield a $26 \%$ lower package temperature using around $12 \%$ less material compared to the lattice-fin design, while maintaining low sensitivity to orientation. Furthermore, they exhibit several defining features and provide insight and general guidelines for the design of passive coolers for LED lamps.
\end{abstract}

Keywords: light-emitting diode, LED lamp, heat sink design, natural convection, passive cooling, topology optimisation

\section{Introduction}

Light-emitting diodes (LEDs) are highly energy-efficient light sources. However, it remains a challenge to adequately cool them since around $70 \%$ of the energy supplied to an LED is converted to heat. This generally leads to high package temperatures, which severely affect the LED light output and lifespan unless sufficiently cooled [1]. Generally, it is necessary to keep the LED package temperature below a given manufacturer-specified temperature, typically around $80^{\circ} \mathrm{C}$.

Above goal is often achieved using forced convection with the help of a small fan. However, the low-power and low-noise benefits of natural convection make it ideal for LED lighting systems in a world with scarce resources. Natural convection inherently does not need an additional energy source forcing the flow, since the temperature differences, caused by the heated LED lamp, cause the air to circulate. Thus, the cooling of the LED lamp is free, in the sense that the energy already supplied to the system is reused to provide the cooling.

Recently, there has been a lot of attention on the design of efficient heat sinks for LED applications in the heat transfer community, focusing mainly on variants of the classical pin or straight-fin heat sinks. Most of this work has been focused on performance for LED lamps in a downwards/vertical orientation, e.g.: Yu et al. [2] investigate various fin configurations for radial heat sinks; Jang et al. [3] perform multidisciplinary optimisation of pin-fin radial heat sinks and found that the pin-fin configuration lead to more uniform cooling performance due

\footnotetext{
${ }^{*}$ Corresponding author

Email address: joealex@mek.dtu.dk (Joe Alexandersen)
}

to repeated leading-edge effects; Jang et al. [4] examine pinfin radial heat sinks with different fin-height profiles and conclude that the fin height should be largest at the circumference and decrease towards the centre. The effect of orientation is less well investigated, but strong examples are: Jang et al. [5] show that the orientation effect for a cylindrical radial straightfin heat sink is strong and find drag and convection efficiency to be inversely correlated; Shen et al. [6] investigate the orientation effect for rectangular straight-fin heat sinks and conclude that denser heat sinks are more sensitive to orientation; Li et al. [7] examine the effect of adding an outer chimney-rim to radial heat sinks, as well as the orientation effect on thermal performance, and concluded that a chimney can increase thermal performance by up to $20 \%$.

The motivation for this work is the design of efficient and visually-pleasing passive coolers for LED lamps. From an industrial design perspective, LEDs offer a large degree of design freedom since LED units are generally quite small and the passive cooling elements have the opportunity to make up the majority of the full lamp design as illustrated by figure 1 . Project partners AT Lighting ApS specialise in the design of LED lighting concepts combining function, aesthetics and additive manufacturing. Based on designer intuition and aesthetics, tapered lattice structures have been proposed to effectively conduct the heat away from the base to the through-flowing air, as well as providing redundant conduction and flow pathways ensuring performance independent of orientation.

The goal of this paper is to investigate whether machine can beat man. Topology optimisation is used to fully utilise the large design freedom provided by the above functional design concept and additive manufacturing. Topology optimisation is 

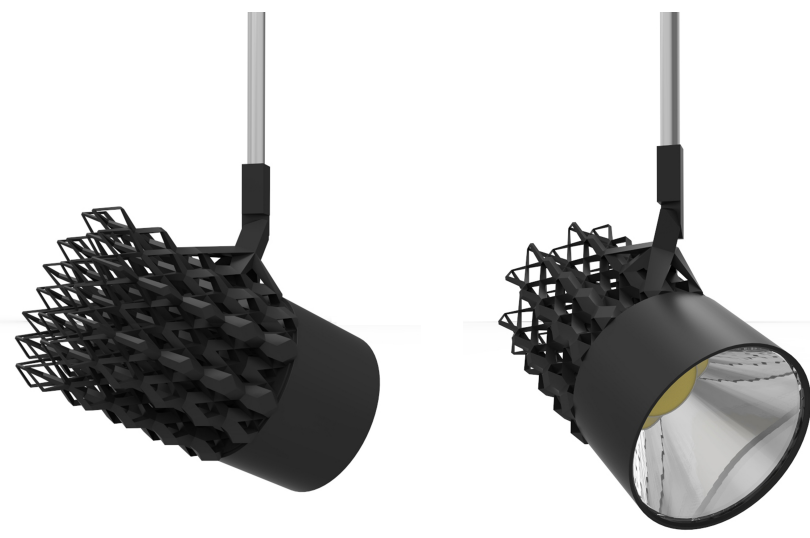

Figure 1: Design concept of a LED spot with an additively-manufactured aluminium heat sink for passive cooling. Pictures are courtesy of AT Lightning ApS.

a material distribution method that originated within structural mechanics $[8,9,10]$ and is used to optimise the layout of a structure with respect to a performance measure under certain design and performance requirements. Topology optimisation allows for a vastly expanded design space compared to classical optimisation techniques, such as shape and size optimisation, as it is not restricted to having an a priori determined initial design and, thus, allows for the appearance of non-intuitive designs.

Topology optimisation for fluid systems began with the treatment of Stokes flow by Borrvall and Petersson [11] and has since been applied to Navier-Stokes flow [12], as well as passive transport problems $[13,14]$, reactive flows [15], transient flows [16, 17, 18], fluid-structure interaction [19, 20], amongst many others. The extension of topology optimisation to turbulent fluid flow is still in its infancy [21,22].

Conjugate heat transfer was originally treated in $[23,24]$ and is a very active field of research today [25, 26, 27, 28, 29]. Most work focuses on forced convection, where the fluid flow is induced by a fan, pump or pressure-gradient. In contrast, the authors have previously presented a density-based topology optimisation approach for both two-dimensional [30] and three-dimensional [31] natural convection problems. A levelset method for steady-state and transient natural convection problems was presented by Coffin and Maute [32]. Recently, a density-based method based on a simplified convection model was presented for plane extruded structures [33] under natural convection. Although, well-performing structures are obtained using this approach, this cannot be guaranteed in general due to the simplified modelling [31, 34].

This paper builds on the large-scale parallel topology optimisation framework previously presented in [31]. Initial results applying the framework to the design of heat sinks for LED lamps was presented in [35]. Major changes to the problem setup have since been made and will be presented herein, along with extensive numerical studies using COMSOL Multiphysics v5.2a [36]. The numerical studies have been validated experimentally through comparisons of an optimised design to two reference designs, but this will be presented separately in [37].

The layout of the paper is as follows: Section 2 briefly outlines the governing equations; Section 3 describes the problem setup and summarises the numerical implementation; Section 4 presents optimised designs and discusses their performance using extensive parameter studies; Section 5 discusses the design features of the optimised designs and attempts to provide general design guidelines for natural convection heat sinks; and Section 6 concludes the findings of the paper. Nomenclature is summarised in Appendix A.

\section{Governing equations}

In order to facilitate the topology optimisation of conjugate natural convective heat transfer between a solid and a surrounding fluid, the equations are posed in a unified domain, $\Omega=\Omega_{f} \cup \Omega_{s}$, where $\Omega_{f}$ is the fluid domain and $\Omega_{s}$ is the solid domain. The subdomain behaviour is achieved through the control of coefficients.

The following unified equations are the Navier-Stokes and convection-diffusion equations under the assumption of: constant fluid properties; incompressible fluid; steady and laminar flow; ${ }^{1}$ neglecting viscous dissipation; a Brinkman porosity term; and the Boussinesq approximation introducing natural convection due to density variations.

$\forall \mathbf{x} \in \Omega$ :

$$
\begin{aligned}
\rho_{0} u_{j} \frac{\partial u_{i}}{\partial x_{j}}-\mu \frac{\partial}{\partial x_{j}}\left(\frac{\partial u_{i}}{\partial x_{j}}+\frac{\partial u_{j}}{\partial x_{i}}\right)+\frac{\partial p}{\partial x_{i}} & =-\alpha(\mathbf{x}) u_{i}-\rho_{0} \beta\left(T-T_{0}\right) g_{i} \\
\frac{\partial u_{j}}{\partial x_{j}} & =0 \\
\rho_{0} c_{p} u_{j} \frac{\partial T}{\partial x_{j}}-\frac{\partial}{\partial x_{j}}\left(k(\mathbf{x}) \frac{\partial T}{\partial x_{j}}\right) & =Q(\mathbf{x})
\end{aligned}
$$

where $\alpha(\mathbf{x})$ is the spatially-varying Brinkman impermeability, $k(\mathbf{x})$ is the spatially-varying thermal conductivity, and $Q(\mathbf{x})$ is the volumetric heat source term.

Theoretically, the impermeability, $\alpha(\mathbf{x})$, is defined as:

$$
\alpha(\mathbf{x})= \begin{cases}0 & \text { if } \mathbf{x} \in \Omega_{f} \\ \infty & \text { if } \mathbf{x} \in \Omega_{s}\end{cases}
$$

in order to ensure zero velocities inside the solid domain. However, numerically this requirement must be relaxed by introducing a finite $\alpha$ in $\Omega_{s}$, as discussed further in e.g. [31].

The thermal conductivity, $k(\mathbf{x})$, is defined as:

$$
k(\mathbf{x})= \begin{cases}k_{f} & \text { if } \mathbf{x} \in \Omega_{f} \\ k_{s} & \text { if } \mathbf{x} \in \Omega_{s}\end{cases}
$$

\footnotetext{
${ }^{1}$ The assumption of steady and laminar flow is verified by the fact that the largest Grashof number (based on the heat sink diameter) for any example in the paper is around $7.3 \times 10^{5}$, which is well below the transition limits for vertical walls and horizontal cylinders $\left(\approx 10^{9}\right)$ [38].
} 

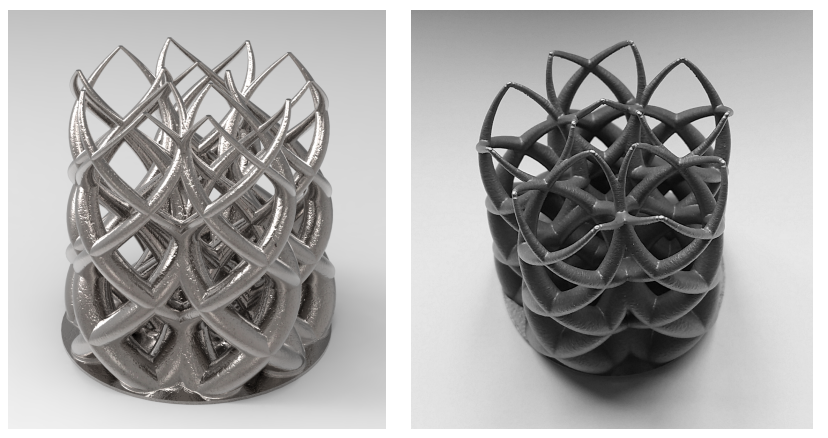

Figure 2: Rendering (left) and additively-manufactured specimen (right) of reference lattice heat sink design supplied by industrial design bureau AT Lighting ApS.

The volumetric heat source term, $Q(\mathbf{x})$, is only active within a predefined subdomain, $\omega \subset \Omega_{s}$, representing the LED package:

$$
Q(\mathbf{x})=\left\{\begin{array}{cc}
0 & \text { if } \mathbf{x} \notin \omega \\
Q_{0} & \text { if } \mathbf{x} \in \omega
\end{array}\right.
$$

In order to facilitate topology optimisation, a continuous design field, $\gamma(\mathbf{x})$, is introduced varying between 0 and 1 , with fluid represented by $\gamma(\mathbf{x})=1$ and solid by $\gamma(\mathbf{x})=0$. The impermeability and conductivity coefficients are linked to this design field making them implicitly spatially-dependent through the design field: $\alpha(\gamma(\mathbf{x}))$ and $k(\gamma(\mathbf{x}))$. Further information on topology optimisation through the control of coefficients and how they specifically are interpolated for this application can be found in [31].

\section{Problem setup}

As briefly discussed in the introduction, the problem at hand is to design efficient and visually-pleasing passive heat sinks for LED lamps as illustrated by figure 1. This Section introduces the reference design, the design problem and the models used for optimisation and orientation studies.

\subsection{Reference design}

Figure 2 shows the reference lattice design supplied by project partners AT Lighting ApS, industrial designers specialised in LED lamps based on passive cooling. The design is one attempt at providing an aesthetically-pleasing, yet functional and efficient, passive heat sink for LED lamps based on designers' intuition. The design is made of a lattice structure, where the thickness is largest at the base and gets thinner further from the heat source, since the amount of heat conducted in the fin decreases along the fin as the heat is convected to the air. Furthermore, the lattice structure is thought to provide plenty of redundant paths for the heat to be conducted through, as well as several flow paths, to provide cooling that is independent of orientation. Lastly, the lattice structure provides for easily additively-manufactured solutions without any support material.

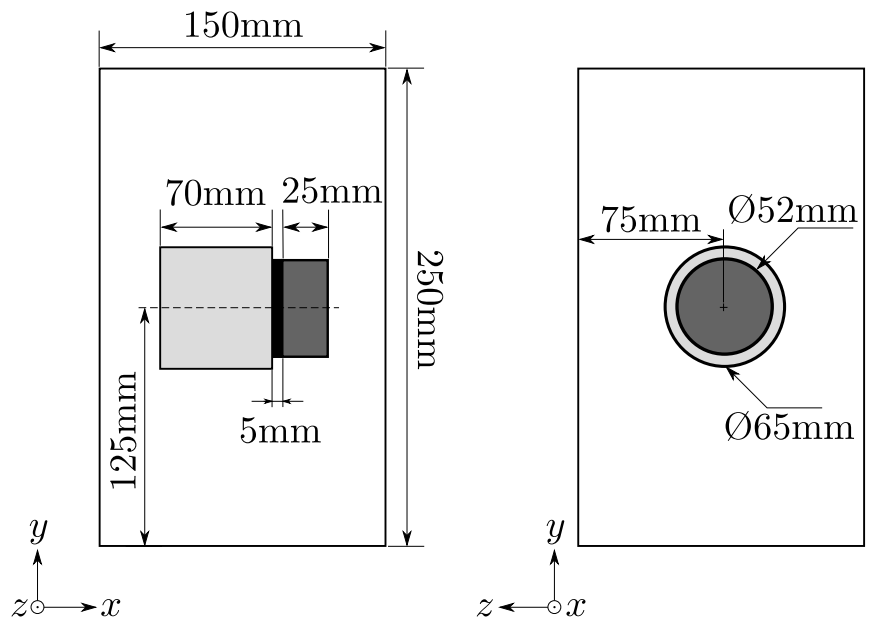

Figure 3: Model setup for the topology optimisation process at the horizontal orientation.

The design is thoroughly compared to designs produced using topology optimisation in the whole of Section 4 and the sensitivity of the lattice performance to orientation is specifically investigated in Section 4.2.

\subsection{Design problem}

As the efficiency and life-time of an LED package is directly correlated to its temperature, the objective of the design problem is to minimise the average temperature of the LED package. In order to make a fair comparison with reference lattice designs, a constraint on the volume of aluminium used for the design is imposed. Although not always necessary nor active for convection-dominated problems, the volume constraint generally helps the design to converge to well-defined topologies.

For further details and a formal mathematical description of the optimisation problem, please see $[35,31]$.

\subsection{Model setup}

To ensure consistency from optimisation model, through performance verification, to experimental validation, the numerical models have been iteratively designed along with the experimental setup used to validate the investigations [37]. In order to resemble the physical situation illustrated in figure 1, but allow for a simple and fully controllable setup, the LED package is emulated using a controllable resistance-heating element. This element is attached to a circular aluminium base plate, in order to spread the heat across the base of the attached heat sinks. The heating element is encased in foam insulation in order to ensure that most of the heat is directed through the heat sinks.

\subsubsection{Optimisation framework}

The optimisation framework used is described in [35, 31], where all details on the numerical implementation and performance are given in details.

In order to simplify the implementation and to save computational resources, a low-fidelity model is introduced for the topology optimisation process, as shown in figure 3 . Instead of modelling all details, the input power, $P$, is applied directly 
in the aluminium base plate (black) as a uniformly distributed volumetric heat source, $Q_{0}=\frac{P}{|\omega|}$. The insulation (dark grey) is then placed below and the heat sink design domain (light grey).

All outer boundaries have open-flow conditions, $\sigma_{i j} n_{j}=0$, except the lower boundary where zero velocities, $u_{i}=0$, are imposed $^{2}$. For the temperature field, all boundaries are set to the ambient temperature, $T_{0}=23.5^{\circ} \mathrm{C}$, except for the upper boundary where a zero diffusive flux, $-k \frac{\partial T}{\partial x_{i}} n_{i}=0$, is imposed. Inside the heat source and insulation domains, the fluid velocity is set strongly to 0 .

For the horizontal orientation, only half of the domain is modelled due to symmetry across the $z$-midplane. For the vertical orientation, the lamp setup is rotated 90 degrees around the mid-point of the outer computational domain and only a quarter of the domain is modelled due to symmetry across the $x$ - and z-midplanes.

The computational domain is discretised using $384 \times 640 \times$ 192 and $192 \times 640 \times 192$ regular cubic elements for the horizontal and vertical case, respectively. This high resolution is needed to allow for small features, of a comparable size to that of the reference lattice design, to form during the optimisation process. Using the above discretisation, the element size is $0.39 \mathrm{~mm}$, which allows for a minimum design length scale ${ }^{3}$ of around $1.95 \mathrm{~mm}$. This is very close to the thinnest member diameter of the reference lattice design.

However, this high resolution comes at a high computational cost. The final problems have a total of 238.1 million and 119.4 million degrees of freedom in the state solution for the horizontal and vertical case, respectively. This currently requires high-performance computing, where a full optimisation procedure (500-1000 design iterations) for the above problems takes about 2-5 full days using 1000-2500 cores on a cluster. This is resource intensive, but the design performance increase and design feature insight generated by topology optimisation is well worth the cost. To make this technology more broadly available and applicable, future work will focus on decreasing this cost using simplified flow models and locally-adapted meshes.

\subsubsection{COMSOL simulations}

COMSOL is used for a parametric study of the optimised geometries. These results are validated against experiments in [37]. As COMSOL allows for locally-adapted meshing, a highfidelity model can be used with low-level details for better accuracy, as shown in figure 4.

Here the full experimental setup is modelled, with the cuboidal heating element $(12 \mathrm{~mm} \times 12 \mathrm{~mm} \times 25 \mathrm{~mm})$ attached to the base plate $(5 \mathrm{~mm} \times \varnothing 52 \mathrm{~mm})$, screws $(25 \mathrm{~mm} \times \varnothing 2.5 \mathrm{~mm})$ connecting the design to the base plate, and copper electrical wires $(\varnothing 3 \mathrm{~mm})$ connected to the heating element. In this model, the input power is distributed in the cuboidal heating element as a volumetric heat source.

\footnotetext{
${ }^{2}$ This is done for two reasons. Firstly, due to a table being present in the experimental setup. Secondly, due to it having a stabilising effect on the steadystate solver in the optimisation framework. It happens that the solver converges to non-physical solutions, if this is left out.

${ }^{3}$ This is due to the applied filtering scheme, where the filter diameter is 2.5 times the element size [31,37]
}

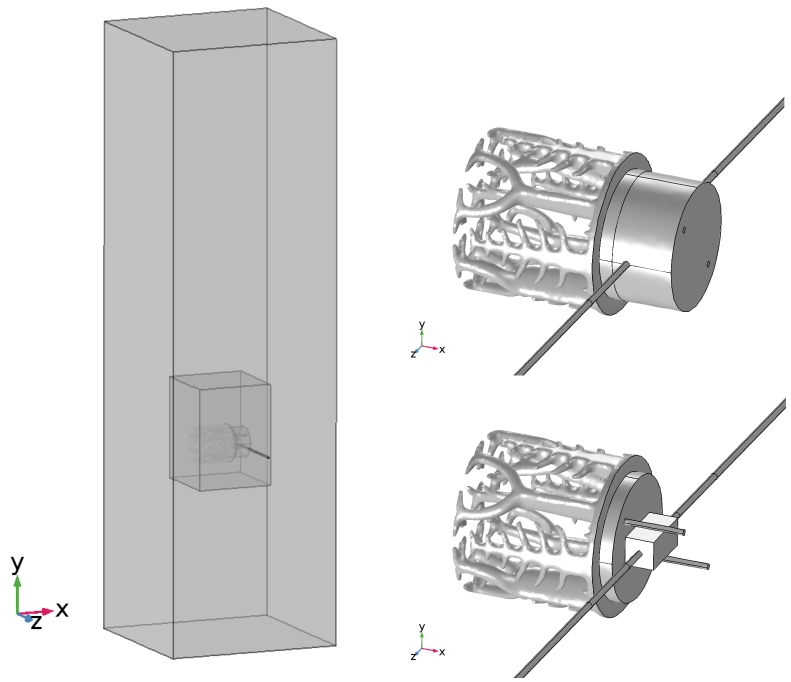

Figure 4: Model setup for the parameter studies in COMSOL with further details.

The boundary conditions are as described above, except noflow conditions are imposed strongly on all outer surfaces of the solid domains. The high-fidelity model is solved for the entire domain using COMSOLs transient flow solver in order to allow for any unstable or asymmetric behaviour to show itself. However, no such behaviour was observed and the fields converged to a steady state.

The mesh is locally-adapted in order to save computational resources for the vast parameter study, while ensuring sufficient accuracy. The outer flow domain uses coarse elements, whereas the mesh is refined inside the volume in the proximity of the lamp, as seen in figure 4 . The mesh gets gradually finer closer to the heat sink geometry, with the heat sink and setup also adaptively-meshed according to geometric detail. A convergence study has been performed for a representative geometry and it was found that a resolution with around 600 thousand tetrahedral elements provided satisfying balance between accuracy and computational time.

\subsubsection{Size of external flow domain}

To sufficiently model the LED lamp in free suspension, the computational flow domain must be large enough to keep boundary effects to a minimum. However, the computational flow domain must also be small enough to provide a realistic computational cost.

The latter is especially important for the topology optimisation framework, which is currently restricted to structured meshes with constant element size. Initial investigations were performed into the sensitivity of flow solution and optimised designs, using relatively coarse meshes for a variety of domain sizes. A domain size of $150 \mathrm{~mm} \times 150 \mathrm{~mm} \times 250 \mathrm{~mm}$ was finally chosen, providing a balance between solution accuracy and computational speed.

Again, with COMSOLs unstructured meshing, the domain could be significantly enlarged without much effect on computational speed. A domain size of $300 \mathrm{~mm} \times 300 \mathrm{~mm} \times 1050 \mathrm{~mm}$ 


\begin{tabular}{c|ccc} 
& $k$ & $\rho$ & $c_{p}$ \\
& {$\left[\mathrm{~W} \cdot \mathrm{m}^{-1} \cdot \mathrm{K}^{-1}\right]$} & {$\left[\mathrm{kg} \cdot \mathrm{m}^{-3}\right]$} & {$\left[\mathrm{J} \cdot \mathrm{kg}^{-1} \cdot \mathrm{K}^{-1}\right]$} \\
\hline Air & 0.026 & 1.2 & 1003 \\
Aluminium & 175 & 2702 & 903 \\
Copper wire & 287 & 8800 & 376 \\
Insulation & 0.026 & 1.2 & 1003
\end{tabular}

Table 1: Material properties used in computational models.

was finally chosen, being large enough to have negligible boundary effects.

\subsection{Material properties}

Table 1 lists material properties used in the computational models. The dynamic viscosity of air is $\mu=1.86 \times 10^{-5} \mathrm{~Pa} \cdot \mathrm{s}$ and the coefficient of volumetric expansion of air is $\beta=3.34 \times$ $10^{-3} \mathrm{~K}^{-1}$. The thermal conductivity of the aluminium may seem relatively low, but it is an estimate of the conductivity of the additively-manufactured aluminium structures used for the experimental validation [37].

\section{Optimised results and performance studies}

Topology optimisation is carried out for a single input power, $P=1.08 \mathrm{~W}$. The following orientation studies are carried out at both $P=1.08 \mathrm{~W}$ and $P=5.26 \mathrm{~W}$, to see if the trends remain the same at higher input power. The motivation for the specific numbers are due to the iterative design of the numerical and experimental setup [37], where these specific values were possible using the chosen power controller.

\subsection{Optimised designs}

Topology optimisation is carried out for two orientations of the lamp: horizontal and vertical. For the horizontal case, two degrees of symmetry are imposed to investigate the influence of symmetry on design features and performance: 1/2th symmetry using a single $z$-symmetry plane and $1 / 8$ th symmetry using three additional symmetry planes rotated $\left\{45^{\circ}, 90^{\circ}, 135^{\circ}\right\}$ around the lamp mid-axis. For the vertical case, only the $1 / 8$ th symmetry case is applied.

Figure 5 shows renderings of the optimised heat sink designs obtained for the various conditions ${ }^{4}$. The optimised designs are organic tree-like structures with main branches extending to secondary and tertiary branches with decreasing thickness, as is well-known for thermal problems [39, 31]. The designs, for the different degrees of symmetry and orientations, have many similarities yet also significant differences. These will be explored in detail in Section 5.

\footnotetext{
${ }^{4}$ Videos showing the designs under rotation are included as supplemental material.
}
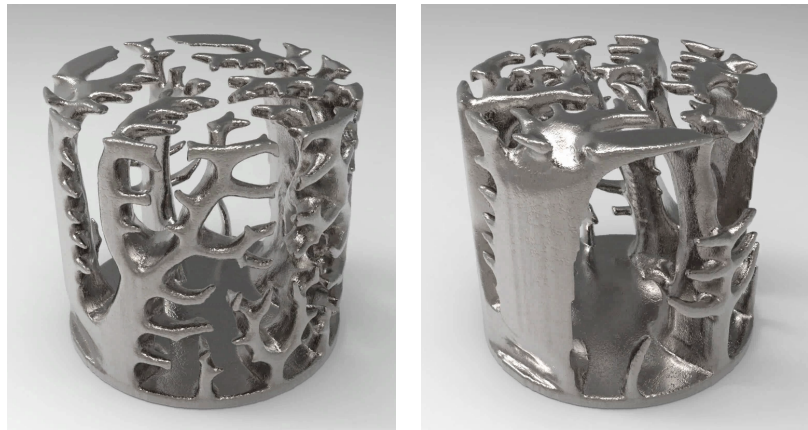

(a) Horizontal - 1/2th symmetry
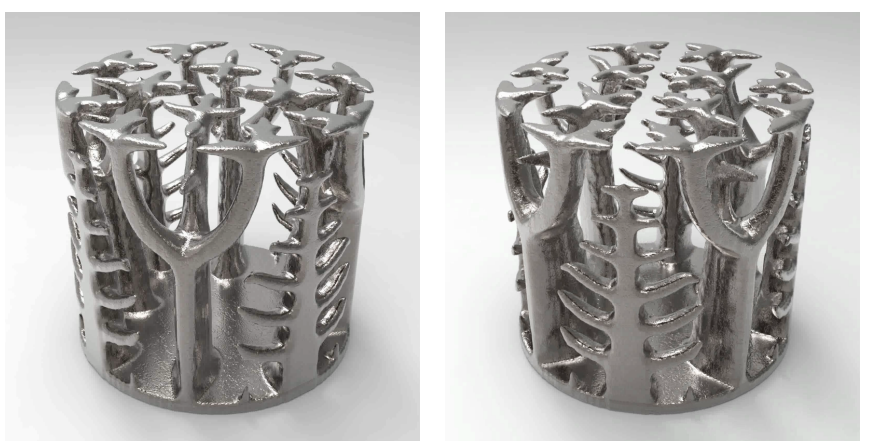

(b) Horizontal - $1 / 8$ th symmetry
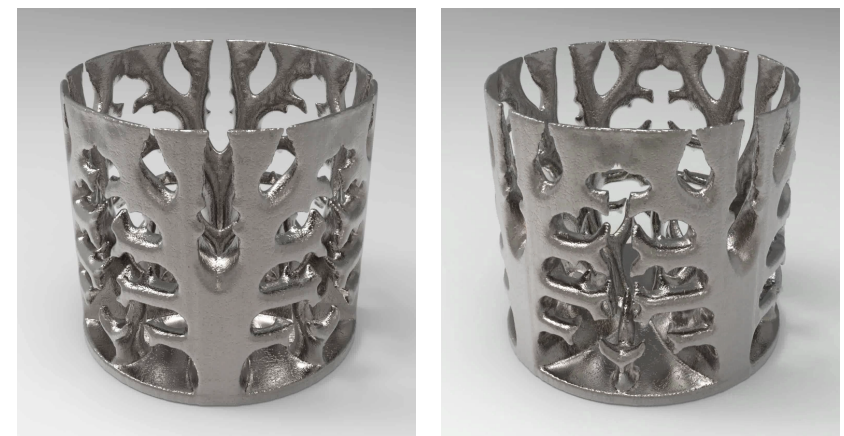

(c) Vertical - $1 / 8$ th symmetry

Figure 5: Renderings of the various optimised heat sink designs. Videos showing the designs under rotation are included as supplementary material. 


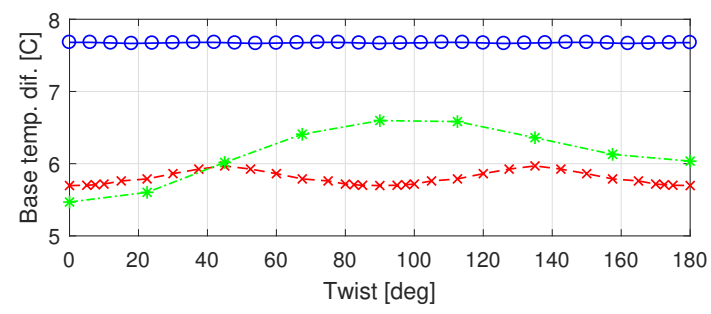

(a) $P=1.08 \mathrm{~W}$

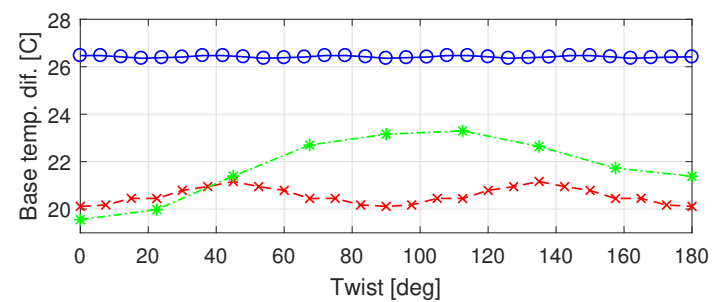

(b) $P=5.26 \mathrm{~W}$

Figure 6: Twist-dependence of the base temperature difference for the three heat sink designs in the horizontal orientation.

\subsection{Orientation studies}

The orientation-dependence of the designs are investigated at the two input power levels. Two types of angular-dependence are investigated, orientation $(\theta)$ and twist $(\eta)$. Orientation varies from vertical $\left(\theta=0^{\circ}\right)$ to horizontal $\left(\theta=90^{\circ}\right)$. For the vertical design the orientation axis goes through the symmetry plane that has a side opening near the base plate. Twist is turning the design around its mid-axis in the horizontal orientation, going from $\eta=0^{\circ}$ to $\eta=180^{\circ}$.

\subsubsection{Twist - effect of increasing symmetry}

Figure 6 shows the dependence of the base temperature difference $\Delta T_{b}$ (base plate temperature relative to ambient temperature) on twist for the three heat sink designs in the horizontal orientation. It can be seen that the performance of the lattice design (AT design) is almost insensitive to twist, due to its compact and repetitive lattice structure. The topology optimised designs are more sensitive, but it is clearly seen that increasing the symmetry (from $1 / 2$ th to $1 / 8$ th) yields a significantly less sensitive performance, from a range of $1.13^{\circ} \mathrm{C}$ down to only $0.27^{\circ} \mathrm{C}$ for $P=1.08 \mathrm{~W}$ and $3.74^{\circ} \mathrm{C}$ to $1.05^{\circ} \mathrm{C}$ for $P=5.26 \mathrm{~W}$. It is interesting to note, that this insensitivity comes at only a slight loss of performance at the optimised position $\left(\eta=0^{\circ}\right)$ of $0.23^{\circ} \mathrm{C}$ for $P=1.08 \mathrm{~W}$ and $0.56^{\circ} \mathrm{C}$ for $P=5.26 \mathrm{~W}$. Furthermore, although optimised for $\eta=0^{\circ}$, the $1 / 2$ th symmetric design performs fairly well when flipped upside down to $\eta=180^{\circ}$, although at an increase of $0.58^{\circ} \mathrm{C}$ for $P=1.08 \mathrm{~W}$ and $1.96^{\circ} \mathrm{C}$ for $P=5.26 \mathrm{~W}$.

It is also worth noting that the change in power from $P=$ $1.08 \mathrm{~W}$ to $P=5.26 \mathrm{~W}$ (a factor of 4.9 ), for all designs and angles, gives a relative change of $\Delta T_{b}$ that is close 3.5. This is in good agreement with typical correlations for laminar free convection where the heat flux scales with the temperature difference to a power of 1.25 [40].

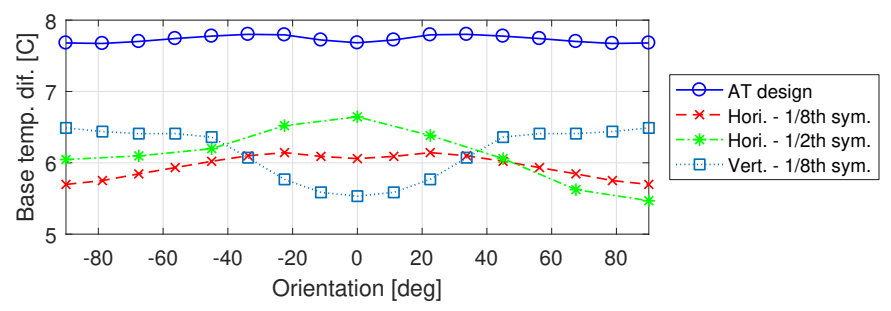

(a) $P=1.08 \mathrm{~W}$

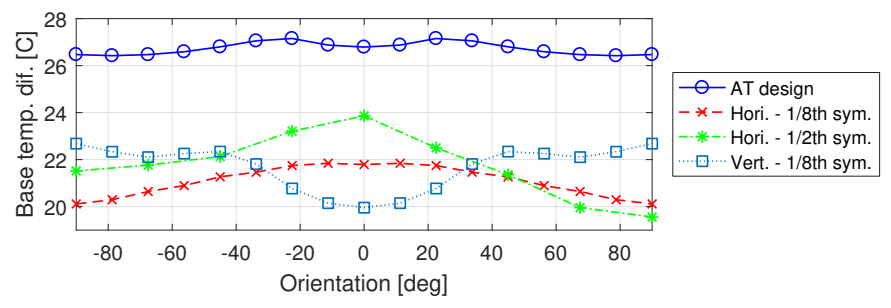

(b) $P=5.26 \mathrm{~W}$

Figure 7: Orientation-dependence of the base temperature difference for all heat sink designs in the vertical orientation.

\subsubsection{Orientation - horizontal versus vertical design}

Figure 7 shows the dependence of the base temperature difference on orientation for all four heat sink designs. The temperature difference again in general scales with a factor close to 3.5 between the two different input powers. As for the twist, it is clear that increasing the symmetry of the heat sink design, decreases the sensitivity to orientation. This is seen by both the lattice design and the $1 / 8$ th symmetric horizontal design. It is once again observed, that the insensitivity gained from increasing the symmetry for the horizontally-optimised design, comes at only a slight loss of performance.

It is interesting to see that optimising for the vertical orientation $(\theta=0)$ yields a significantly lower base temperature (around $0.53^{\circ} \mathrm{C}$ at at $P=1.08 \mathrm{~W}$ ) than for the horizontal design with equivalent symmetry. However, the performance varies quite significantly for the vertical design, when $|\theta|>20^{\circ}$. This suggests that the design features of the vertical design are mainly good for the vertical orientation, whereas the characteristics horizontal design are good in the general case. This will be further explored in Section 5.

\section{Design features}

The design features of the optimised designs are investigated for $P=1.08 \mathrm{~W}$, since this is the design power. Section 5.1 investigates the reference lattice design, the $1 / 2$ th symmetric horizontal design and the 1/8th symmetric horizontal design at the horizontal orientation $\left(\theta=90^{\circ}\right)$. Section 5.2 compares the $1 / 8$ th symmetric vertical design to the reference lattice design and the $1 / 8$ th symmetric horizontal design at the vertical orientation $\left(\theta=0^{\circ}\right)$.

\subsection{Horizontal orientation}

Figure 8 shows the heat sink surface temperature distributions with streamtubes illustrating the natural convective flow 


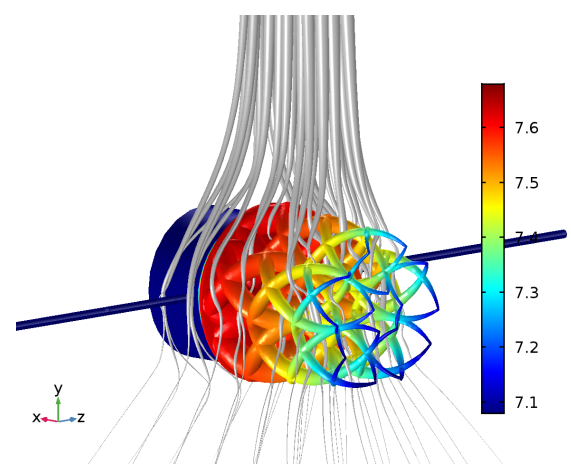

(a) Lattice design

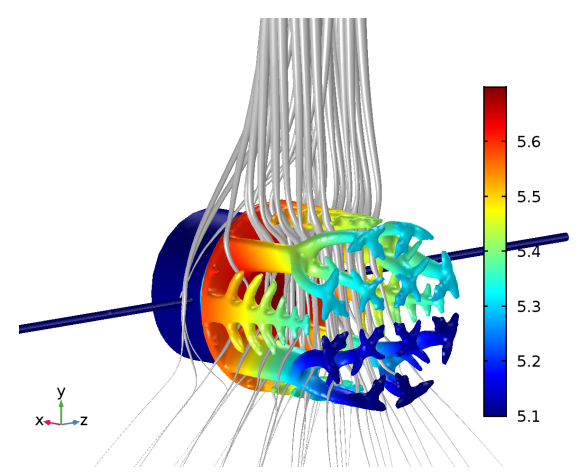

(b) $1 / 8$ th symmetric design

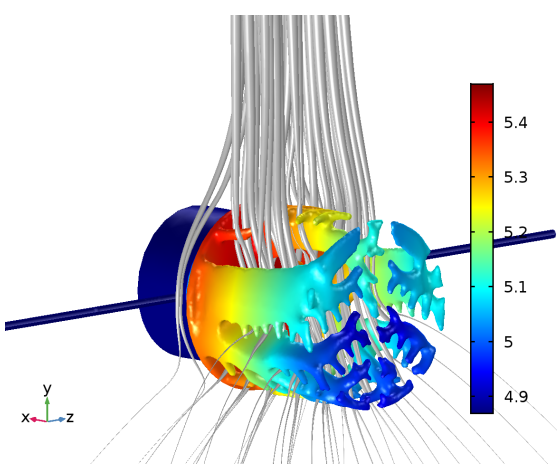

(c) $1 / 2$ th symmetric design

Figure 8: Temperature distribution $\left(\Delta T_{b}\left[{ }^{\circ} \mathrm{C}\right]\right)$ of the heat sink surfaces and streamtubes for the three designs at horizontal orientation $\left(\theta=90^{\circ} \mathrm{C}\right)$. Please note that the scales are different for each design and that the shown is the temperature difference above ambient.

through them. The scales are different for each design, but the range is the same: $\left[\Delta T_{b}-0.6 ; \Delta T_{b}\right]$. This is done so the temperature variation over the designs can be compared. It can be seen that the temperature variation, in the axial direction, in general is more one-dimensional for the lattice design. Whereas for the topology-optimised designs, the temperature generally varies quite significantly in the vertical direction also. This is due to the locally higher flow velocities generated by the optimised designs, which cools the bottom parts more efficiently than for the lattice design. Looking at the flow, it can be seen to be generally more dispersed around the heat sink for the lattice design, yet a more focused plume forms over it. Contrarily, the flow path is more centralised for the optimised designs, with a large cavity in the centre where the flow can accelerate vertically without obstacles, however forming a wider plume above them. The above flow features are investigated further in Section 5.1.2.

\subsubsection{Quantitative comparison}

In order to quantitatively compare the three designs, the following quantities are defined as measures:

- Volume:

$$
V_{h s}=\int_{\Omega_{h s}} d V
$$

where $\Omega_{h s}$ is the heat sink domain.

- Surface area:

$$
A_{h s}=\int_{\Gamma_{h s}} d S
$$

where $\Gamma_{h s}$ is the heat sink surface.

- Surface per volume:

$$
f_{h s}=\frac{V_{h s}}{A_{h s}}
$$

- Buoyancy force:

$$
F_{b}=-\int_{\Omega_{b}} \rho_{0} \beta\left(T-T_{0}\right) g_{2} d V
$$

where $\Omega_{b}$ is a cuboidal volume around the lamp (heat sink, heat source, insulation) that extends $1 / 2$ design radius, $16.25 \mathrm{~mm}$, to all sides.
- Drag force:

$$
F_{d}=\int_{\Gamma_{s f} \cap \Omega_{b}}\left(\mu\left(\frac{\partial u_{2}}{\partial x_{j}}+\frac{\partial u_{j}}{\partial x_{2}}\right)-\tilde{p} \delta_{2 j}\right) n_{j} d S
$$

where $\Gamma_{s f}$ is the solid-fluid interface.

- Resulting force:

$$
F_{r}=F_{b}+F_{d}
$$

- Mass flow:

$$
\dot{m}=\int_{\Gamma_{m f}} \rho_{0} u_{2} d S
$$

where $\Gamma_{m f}$ is a $x z$-plane at 1.5 design radii, $48.75 \mathrm{~mm}$, above the upper edge of the heat sink.

- Average heat transfer coefficient:

$$
\bar{h}=\frac{1}{A_{h s}} \int_{\Gamma_{h s}} \frac{q_{i} n_{i}}{T-T_{0}}
$$

Table 2 shows these quantities calculated for the three designs at the horizontal orientation $\left(90^{\circ}\right)$. It can be seen that the surface area and volume of the heat sinks, as well as the ratio between them, are not correlated to the performance of the heat sink, as quantified by the base temperature difference. This shows that minimising the base temperature is not simply achieved by increasing the heat sink area or the volume of material used. In fact, the optimised design shows a $25 \%$ decrease in $\Delta T_{b}$ for $15 \%$ less material. The resulting force and the generated mass flow are positively correlated with the performance. This makes perfect sense as increasing the total uplift force on the air, increases the mass flow. By increasing the generated mass flow, this in turn increases the convective heat transfer away from the heat sink reflected by the increasing heat transfer coefficient. It is interesting to note, that the buoyancy force generated is inversely correlated with the performance. A higher temperature causes higher density differences and larger buoyancy forces. The drag is also inversely correlated and decreases at a higher rate, leading to a total increase in the resulting uplift force. This suggests that drag plays an important role 


\begin{tabular}{c|ccccccccc} 
Design & $\Delta T_{b}$ & $V_{h s}$ & $A_{h s}$ & $\begin{array}{c}f_{h s} \\
{\left[10^{2} \mathrm{~m}^{-1}\right]}\end{array}$ & $\begin{array}{c}F_{b} \\
{\left[10^{-5} \mathrm{~N}\right]}\end{array}$ & $\begin{array}{c}F_{d} \\
{\left[10^{-5} \mathrm{~N}\right]}\end{array}$ & $\begin{array}{c}F_{r} \\
{\left[10^{-5} \mathrm{~N}\right]}\end{array}$ & $\begin{array}{c}\dot{m} \\
{\left[10^{-4} \mathrm{~kg} \cdot \mathrm{s}^{-1}\right]}\end{array}$ & $\begin{array}{c}\bar{h} \\
{\left[\mathrm{~W} \cdot \mathrm{m}^{-2} \cdot \mathrm{K}^{-1}\right]}\end{array}$ \\
\hline Lattice & 7.68 & 4.46 & 2.55 & 5.72 & 4.99 & -2.57 & 2.42 & 6.94 & 3.81 \\
1/8th sym. & 5.70 & 3.94 & 2.36 & 6.00 & 4.76 & -2.31 & 2.45 & 7.11 & 6.38 \\
1/2th sym. & 5.47 & 4.61 & 2.39 & 5.18 & 4.42 & -1.56 & 2.86 & 7.20 & 6.67
\end{tabular}

Table 2: Quantities calculated for the reference lattice design (AT design) and the two optimised designs. Data evaluated at horizontal orientation (90 $)$.

in the performance of passive coolers and that it must be balanced in a careful trade-off with the buoyancy force. The fact that drag and average heat transfer coefficient is inversely correlated was also observed by Jang et al. for cylindrical radial straight-fin heat sink [5].

\subsubsection{Qualitative comparison}

In order to further explain the performance differences between the various designs, a qualitative comparison is carried out based on the physical fields on and around the heat sink.

\section{Pressure field:}

Figure 9 shows the pressure fields along with in-plane velocity arrows for two planes through the heat sink designs, one near the base and one near the middle. The shown pressure is the augmented pressure field, $\hat{p}=p-\rho_{0} g_{i} x_{i}$, i.e. the local pressure with the local hydrostatic pressure subtracted. The gradient of $\hat{p}$ describes the local driving force in equation (1).

Focusing on the lattice design first (figures 9a and 9b), it can be seen that due to the dense nature of the lattice structure, many stagnation pressure regions are observed when the flow hits the fins. This of course adds to the large drag force observed in table 2. Another interesting feature is that a significant part of the flow is diverted around the heat sink, rather than through it, when comparing to especially the $1 / 8$ th symmetric design (figures 9c and 9d.) The lattice design generates two strong air streams outside the fin area. The air streams from each side meet under an angle above the fin, changing direction to a vertically flowing single plume. This results in a local pressure peak. The optimised designs are more efficient in directing the flow through the geometry and achieve a wider distributed plume.

Moving on to the $1 / 8$ th symmetric design (figures $9 \mathrm{c}$ and $9 \mathrm{~d}$ ) the shape of the elongated fins is clearly seen to be there in order to control and direct the flow, pulling it in at the bottom, directing it towards the middle and away at the top. The large inner cavity allows the flow to accelerate uninhibited and perfectly aligned with the direction of gravity and thus maximising the velocity increase over the design height. This inner cavity seems to compensate for the pressure loss over the lower fins, yielding a large area of close to ambient pressure. Significantly fewer stagnation regions are observed compared to the lattice design.

Finally, for the $1 / 2$ th symmetric design (figures $9 e$ and $9 f$ ), clear aerofoil-like cross-sections are observed: two large main fins near the base, each splitting into two further out, clearly having the purpose of controlling and directing the flow inside the design cavity. It can be seen that large areas of negative pressure are present in the inner cavity, indicating that a strong buoyancy force is acting here creating local flow acceleration.

As discussed above, the lattice design blocks much of the flow through the heat sink and a single narrow plume forms above it. The optimised designs generate more internal flow which is better distributed, yielding better internal heat transfer, a wider plume and higher mass flow.

\section{Temperature field:}

Figure 10 shows the temperature fields for the same two planes as for the pressure. The scales are different for each design, but the range is the same: $\left[\Delta T_{b}-3 ; \Delta T_{b}\right]$, so the temperature variation is comparable.

For the lattice design (figures 10a and 10b), the air temperature inside the heat sink is homogeneous and high compared to the other designs. The air is quickly heated by the initial fins, leading to low heat transfer in the centre and at the top due to the already high air temperature.

In comparison, the $1 / 8$ th symmetric design (figures $10 \mathrm{c}$ and 10d) exhibits significantly cooler air temperatures. It is clear that sufficient amounts of cold air is drawn in at the bottom and directed towards the top to be used to efficiently cool the top fins, which is made clearer by looking at the flow paths in figures $9 \mathrm{c}$ and $9 \mathrm{~d}$. This illustrates the fact, that the cavity not only provides for some form of low resistance / momentum source region to accelerate (or at least not decelerate) the flow, it also has significance with respect to heat transfer. If fins were present in the centre, they would conduct heat out and warm the air before it hit the top. The elongated fins are not only present in order to accelerate and guide the flow. A cylinder and an elongated fin of the same size has approximately the same area of stagnation pressure, however, the elongated fin provides more side area for convective heat transfer.

Very similar observations as above can be made for the $1 / 2$ th symmetric design (figures 10e and 10f). Here it is is especially evident that cold air is allowed to flow in from the bottom to be used at the top away from the base (figure 10f), whereas fins are only placed at the bottom on the base (figure 10e) in order not to have significant hotspots on the baseplate.

\section{Surface heat flux:}

Figure 11 shows the distribution of the heat flux, normal to the heat sink surface, for the three horizontal designs. It can be seen that most of the internal structure of the lattice design generally has a very low heat transfer. This is because the material is inefficiently placed, due to the high air temperatures leading 


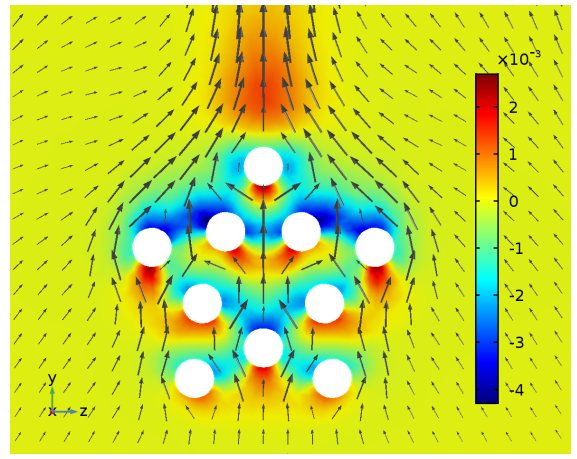

(a) Lattice - base

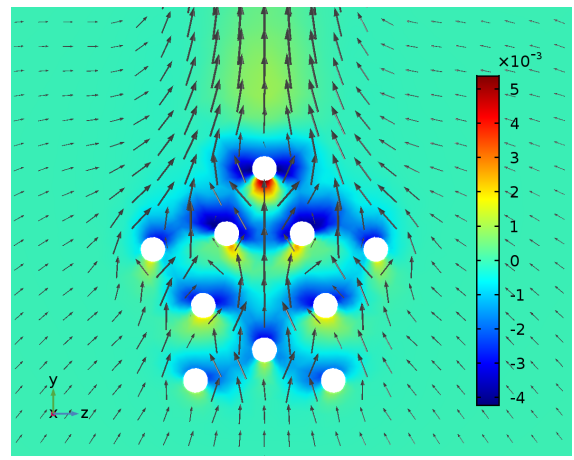

(d) Lattice - mid

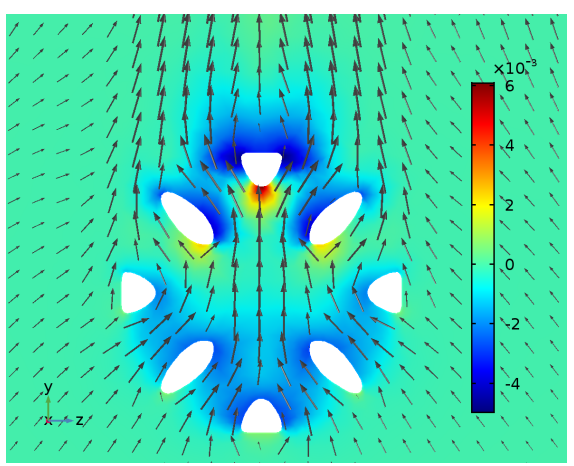

(b) $1 / 8$ th - base

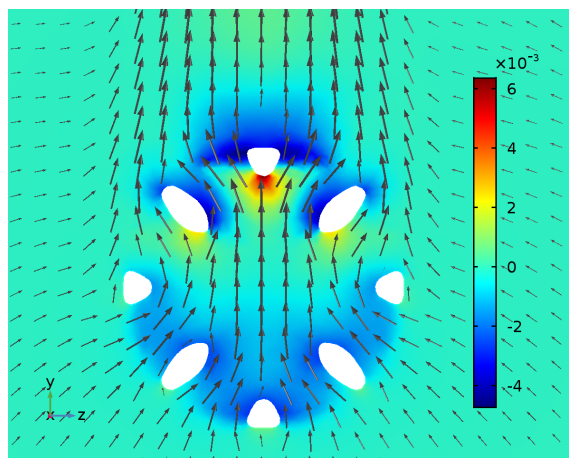

(e) $1 / 8$ th - mid

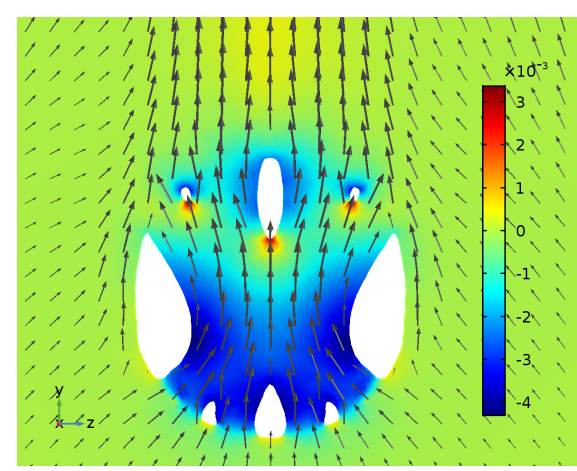

(c) $1 / 2$ th - base

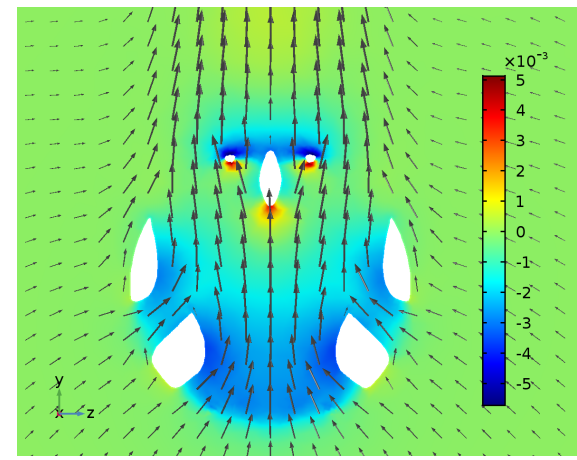

(f) $1 / 2$ th - mid

Figure 9: Augmented pressure fields $(\hat{p}[\mathrm{~Pa}])$ with in-plane velocity arrows near middle and near base of the three designs in horizontal orientation $\left(90^{\circ}\right)$. Please note that the colour scale is different for all plots to show details and the arrows are scaled logarithmically.

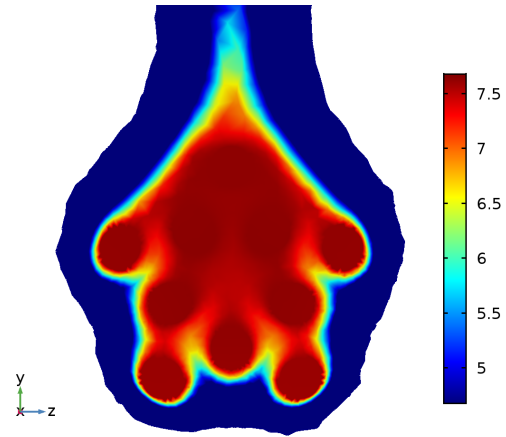

(a) Lattice - base

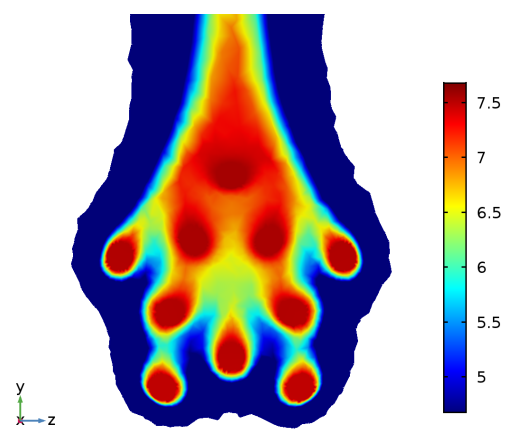

(d) Lattice - middle

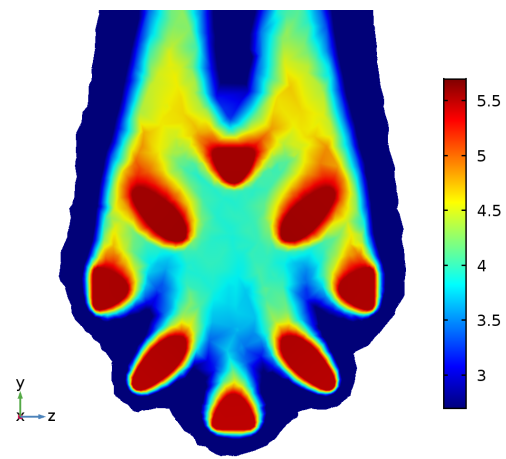

(b) $1 / 8$ th - base

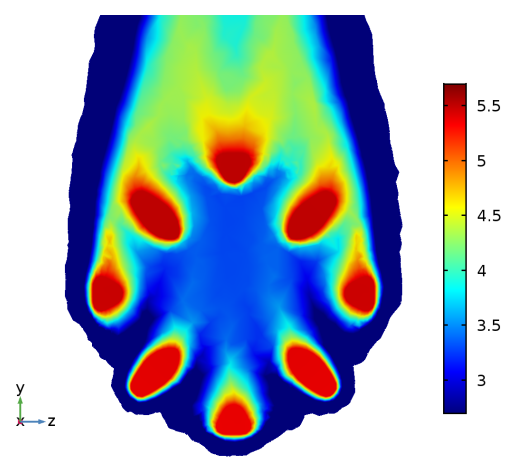

(e) $1 / 8$ th - middle

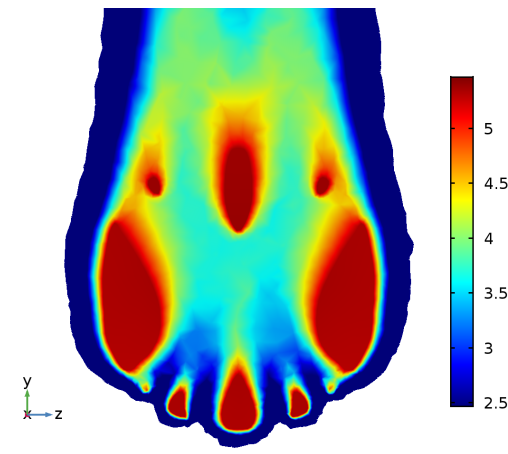

(c) $1 / 2$ th - base

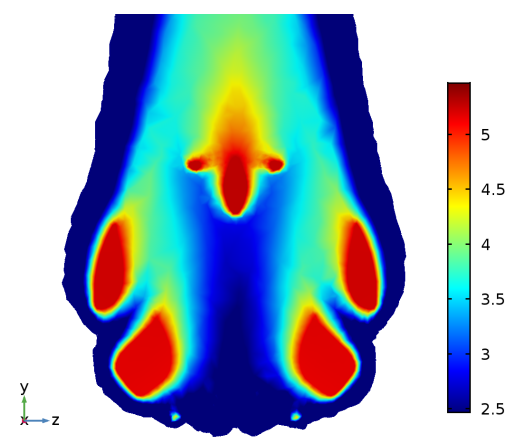

(f) $1 / 2$ th - middle

Figure 10: Temperature fields $\left(\Delta T_{b}\left[{ }^{\circ} \mathrm{C}\right]\right)$ near middle and near base of the three designs in horizontal orientation $\left(\theta=90^{\circ}\right)$. Please note that the scales are different for each design and that colour show the temperature difference from ambient temperature. 


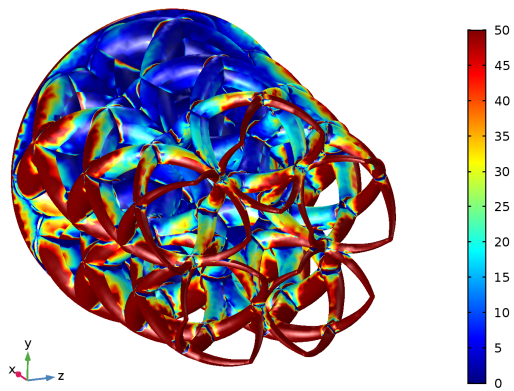

(a) Lattice design

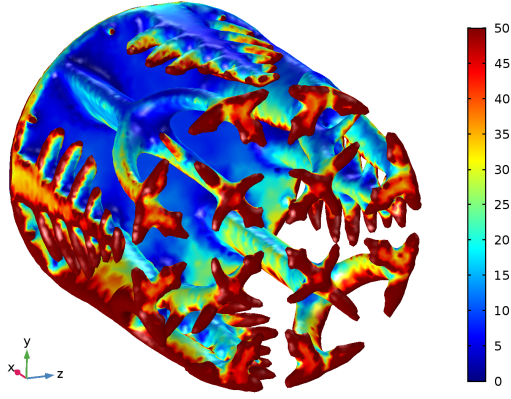

(b) $1 / 8$ th symmetric design

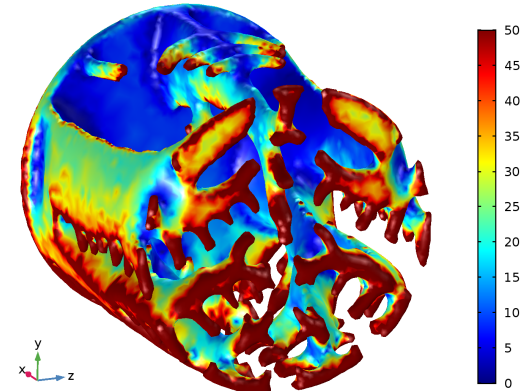

(c) $1 / 2$ th symmetric design

Figure 11: Distributions of the heat sink surface flux $\left(\mathrm{W} \cdot \mathrm{m}^{-2}\right)$ for the three designs in horizontal orientation $\left(\theta=90^{\circ}\right)$.

to low convective heat transfer, as previously discussed. However, for the more open topology-optimised designs, it can be seen that the top fins also have relatively large convection contributions. This is especially the case for the inside surface of the top fins. For all the designs, it can be seen that the largest convective heat transfer is present on the outer surfaces. This is because the surrounding cold air directly hits these surfaces and thus provides yet another reason for concentrating the material around the outer edges of the heat sink domain.

\subsection{Vertical orientation}

Figure 12 shows the temperature distribution of the heat sink surfaces for the three designs at vertical orientation $\left(\theta=0^{\circ}\right)$ : reference lattice design, horizontal design with $1 / 8$ th symmetry, and vertical design with $1 / 8$ th symmetry. Streamtubes are also shown to illustrate the flow through the heat sinks. A significant difference between the vertical and horizontal designs, is the lack of material at the top of the vertical design. This is partly because the material is less efficiently used here, due to the higher temperature of the upcoming air. It is also due to drag considerations, as the open core allows for the air to accelerate uninhibited without being slowed down at the top. This is clearly reflected in the significantly lower drag force, $F_{d}=1.93 \times 10^{-5} \mathrm{~N}$, for the vertical design, compared to $2.59 \times 10^{-5} \mathrm{~N}$ and $2.65 \times 10^{-5} \mathrm{~N}$ for the horizontal and lattice designs, respectively.

Another detail is the fact that connected members are seen at the top of the vertical design. This provides for a chimneylike effect, where the flow is forced inwards and through the design, as seen in figure 12c. This effect is well-documented in the heat transfer literature, see e.g. [7]. Calculating the mass flow into the design ${ }^{5}$, it reveals that it is significantly larger for the vertical design, $1.97 \times 10^{-4} \mathrm{~kg} \cdot \mathrm{s}^{-1}$, compared to the other designs, with $1.60 \times 10^{-4} \mathrm{~kg} \cdot \mathrm{s}^{-1}$ and $1.56 \times 10^{-4} \mathrm{~kg} \cdot \mathrm{s}^{-1}$, for the horizontal and lattice designs, respectively.

A final significant difference between the horizontal and vertical designs, is the presence of fins on the inner part of the

\footnotetext{
${ }^{5}$ That is the integration of the radial velocity on a cylindrical surface circumscribing the designs multiplied by the density.
}

baseplate. This is necessary in order to adequately cool the entire baseplate, as the velocities are relatively low in the centre. Figure 13 illustrates the differences in fin distribution on the baseplate of the two optimised $1 / 8$ th symmetric designs. The radial velocity on a plane just above the baseplate is shown, as well as the surface normal heat flux. It can be seen that more material is distributed over the baseplate: both in the longer extent of the primary fins; as well as a central pointy fin and secondary fins extending from the primary. The left column of figure 13 clearly shows that the radial velocity is significantly larger for the vertical design and more well distributed along the surfaces of the fins, compared to for the horizontal design. This leads to significantly higher convective heat transfer on the baseplate as seen from the right column of figure 13, where very little heat is transfer from the baseplate for the horizontal design. Although significantly more complex, the optimised design for the vertical orientation is reminiscent of the "longmiddle" (LM) configuration [2] and also exhibits lowering fin height towards the centre as observed by Jang et al. [4].

\section{Conclusions}

Natural convection is often the preferred method of cooling small electronic devices like LED lamps. New manufacturing methods allow for more sophisticated designs. It is therefore very relevant to develop new optimisation methods and design guidelines. The objective can be any combination of LED temperature, material properties and volume, dimensions and increasing robustness with respect to orientation and exploitation conditions. The present study demonstrates that it is feasible and beneficial to perform topology optimisation on natural cooling fin geometries and that this leads to new ideas and insights for the design of cooling devices. The findings agree well with the established understanding of cooling fin design, but also offers new ideas and design directions.

\subsection{General design guidelines}

The analysis of the optimised designs can be generalised into the following guidelines: 


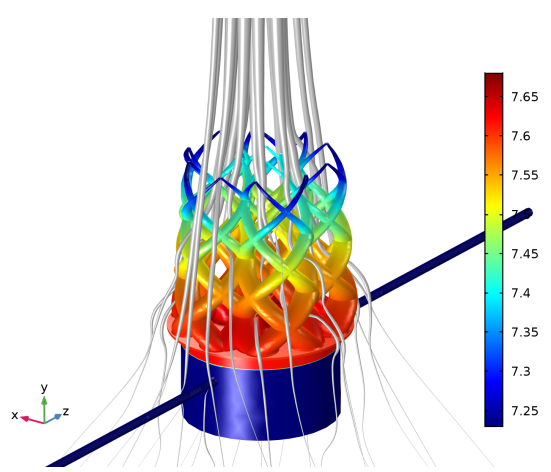

(a) Lattice design

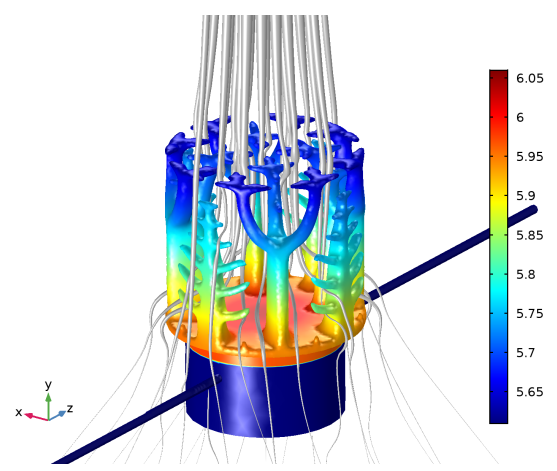

(b) $1 / 8$ th - horizontal

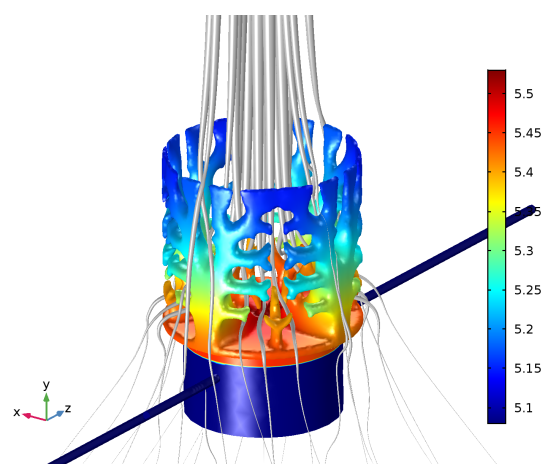

(c) $1 / 8$ th - vertical

Figure 12: Temperature distribution of the heat sink surfaces and streamtubes for three designs at vertical orientation $\left(\theta=0{ }^{\circ} \mathrm{C}\right)$. Please note that the scales are different for each design and that the shown is the temperature difference above ambient.
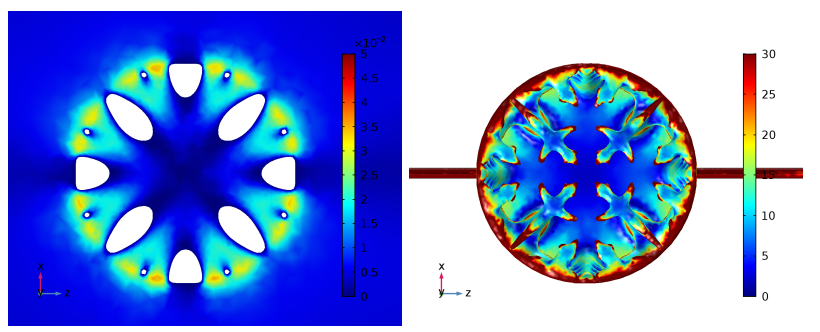

(a) $1 / 8$ th - horizontal
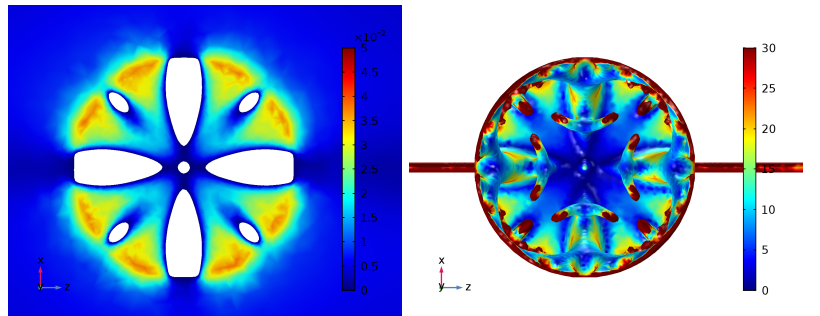

(b) $1 / 8$ th - vertical

Figure 13: Differences in member distribution on the baseplate. Left: radial velocity $\left(\mathrm{m} \cdot \mathrm{s}^{-1}\right)$ on a plane $2 \mathrm{~mm}$ above the baseplate. Right: Surface normal heat flux $\left(\mathrm{W} \cdot \mathrm{m}^{-2}\right)$.
- Making a hollow core by placing most fins at the edges of the design supports higher flow velocities. The higher heat transfer at fins compensates for not having fins at the core.

- Aerofoil-like cross-sections of fins give low drag and allow for higher velocities when aligned with the flow direction. They also have large surface area allowing more heat to be transferred. Such setups can be much more efficient than circular cross sections even if some of the fins are not aligned with the flow.

- A design with rotational symmetries can have efficiency comparable to a cooler optimised for a specific orientation. The solution is robust with respect to multiple exploitation scenarios providing larger on-site freedom and avoiding failures due to errors in the montage.

Results from a topology optimisation, usually have to be "translated" into a simplified geometry that takes manufacturing and practical constraints into account. The guidelines above can be useful in such a process.

\subsection{Future developments}

The presented optimisation has produced performant designs. However, both methods and applications can be developed further:

- The computational cost is very high, and can be reduced in several ways, e.g., by introducing locally adapted meshes, developing optimised application specific solvers.

- The optimisation formulation can be extended further to include multiple load/orientation cases. Although this would increase computational cost, it would result in even more robust and possibly more interesting designs unachievable by simple engineering intuition.

- The optimisation can include constraints linked to different manufacturing processes. An example is not allowing "overhang" features in geometries for 3D-printing, since these presently require support structures and subsequent post-processing. Another important example is accounting for limitations related to casting. 
- The model in the optimisation framework can be extended to include turbulence. This would increase computational cost and introduce uncertainties related to the turbulence, but would allow for a wide range of new applications.

- Another addition to the framework would be the radiation that can play a major role at higher temperature differences than the ones found in the present work. However, it should be pointed out that radiation is far from being trivial to handle in a density-based topology optimisation method.

\section{Acknowledgements}

This work was funded by Innovation Fund Denmark through the HyperCool project, by the Danish Council for Independent Research through the TOpTEn project (DFF-4005-00320), as well as by the Villum Foundation through the NextTop project. The authors would like to thank AT Lighting ApS for their collaboration through the HyperCool project.

\section{Appendix A. Nomenclature}

[1] N. Narendran, Y. Gu, J. Freyssinier, H. Yu, L. Deng, Solid-state lighting: failure analysis of white \{LEDs\}, Journal of Crystal Growth 268 (3-4) (2004) 449 - 456, \{ICMAT\} 2003, Symposium H, Compound Semiconductors in Electronic an d Optoelectronic Applications. doi:10.1016/j.jcrysgro.2004.04.071.

[2] S.-H. Yu, K.-S. Lee, S.-J. Yook, Optimum design of a radial heat sink under natural convection, International Journal of Heat and Mass Transfer 54 (11-12) (2011) 2499 - 2505. doi:10.1016/j.ijheatmasstransfer.2011.02.012.

[3] D. Jang, S.-H. Yu, K.-S. Lee, Multidisciplinary optimization of a pin-fin radial heat sink for \{LED\} lighting applications, International Journal of Heat and Mass Transfer 55 (4) (2012) 515 - 521. doi:10.1016/j.ijheatmasstransfer.2011.11.016.

[4] D. Jang, S.-J. Yook, K.-S. Lee, Optimum design of a radial heat sink with a fin-height profile for high-power $\{$ LED $\}$ lighting applications, Applied Energy 116 (2014) 260 - 268. doi:10.1016/j.apenergy.2013.11.063.

[5] D. Jang, S.-J. Park, S.-J. Yook, K.-S. Lee, The orientation effect for cylindrical heat sinks with application to \{LED\} light bulbs, International Journal of Heat and Mass Transfer 71 (2014) 496 - 502. doi:10.1016/j.ijheatmasstransfer.2013.12.037.

[6] Q. Shen, D. Sun, Y. Xu, T. Jin, X. Zhao, Orientation effects on natural convection heat dissipation of rectangular fin heat sinks mounted on \{LEDs\}, International Journal of Heat and Mass Transfer 75 (2014) 462 469. doi:10.1016/j.ijheatmasstransfer.2014.03.085.

[7] B. Li, Y.-J. Baik, C. Byon, Enhanced natural convection heat transfer of a chimney-based radial heat sink, Energy Conversion and Management 108 (2016) 422 - 428. doi:10.1016/j.enconman.2015.11.037.

[8] M. P. Bendsøe, O. Sigmund, Topology Optimization: Theory, Methods and Applications, Springer, 2003, ISBN: 3-540-42992-1.

[9] O. Sigmund, K. Maute, Topology optimization approaches, Structural and Multidisciplinary Optimization 48 (6) (2013) 1031-1055. doi:10.1007/s00158-013-0978-6.

[10] J. D. Deaton, R. V. Grandhi, A survey of structural and multidisciplinary continuum topology optimization: post 2000, Structural and Multidisciplinary Optimization 49 (1) (2013) 1-38. doi:10.1007/s00158-013-0956$\mathrm{z}$.

[11] T. Borrvall, J. Petersson, Topology optimization of fluids in Stokes flow, International Journal for Numerical Methods in Fluids 41 (1) (2003) 77107. doi:10.1002/fld.426.

[12] A. Gersborg-Hansen, O. Sigmund, R. Haber, Topology optimization of channel flow problems, Structural Multidisciplinary Optimization 30 (3) (2005) 181-192. doi:10.1007/s00158-004-0508-7.
[13] C. S. Andreasen, A. R. Gersborg, O. Sigmund, Topology optimization of microfluidic mixers, International Journal for Numerical Methods in Fluids 61 (5) (2009) 498-513. doi:10.1002/fld.1964.

[14] D. Makhija, K. Maute, Level set topology optimization of scalar transport problems, Structural and Multidisciplinary Optimization 51 (2) (2015) 267-285. doi:10.1007/s00158-014-1142-7.

[15] F. Okkels, H. Bruus, Scaling behavior of optimally structured catalytic microfluidic reactors, Phys. Rev. E 75 (1) (2007) 016301. doi:10.1103/PhysRevE.75.016301.

[16] S. Kreissl, G. Pingen, K. Maute, Topology optimization for unsteady flow, International Journal for Numerical Methods in Engineering 87 (13) (2011) 1229-1253. doi:10.1002/nme.3151.

[17] Y. Deng, Z. Liu, P. Zhang, Y. Liu, Y. Wu, Topology optimization of unsteady incompressible Navier-Stokes flows, Journal of Computational Physics 230 (17) (2011) 6688-6708. doi:10.1016/j.jcp.2011.05.004.

[18] S. Nørgaard, O. Sigmund, B. Lazarov, Topology optimization of unsteady flow problems using the lattice boltzmann method, Journal of Computational Physics 307 (2016) 291 - 307. doi:10.1016/j.jcp.2015.12.023.

[19] G. H. Yoon, Topology optimization for stationary fluid-structure interaction problems using a new monolithic formulation, International Journal for Numerical Methods in Engineering 82 (5) (2010) 591-616. doi:10.1002/nme.2777.

[20] N. Jenkins, K. Maute, Level set topology optimization of stationary fluid-structure interaction problems, Structural and Multidisciplinary Optimization 52 (1) (2015) 179-195. doi:10.1007/s00158-015-1229-9.

[21] E. A. Kontoleontos, E. M. Papoutsis-Kiachagias, A. S. Zymaris, D. I. Papadimitriou, K. C. Giannakoglou, Adjoint-based constrained topology optimization for viscous flows, including heat transfer, Engineering Optimization 45 (8) (2013) 941-961. doi:10.1080/0305215X.2012.717074.

[22] C. B. Dilgen, S. B. Dilgen, D. R. Fuhrman, O. Sigmund, B. S. Lazarov, Topology optimization of turbulent flows, in review.

[23] E. Dede, Multiphysics topology optimization of heat transfer and fluid flow systems, in: Proceedings of the COMSOL Conference 2009 Boston, 2009.

[24] G. H. Yoon, Topological design of heat dissipating structure with forced convective heat transfer, Journal of Mechanical Science and Technology 24 (6) (2010) 1225-1233. doi:10.1007/s12206-010-0328-1.

[25] J. H. K. Haertel, K. Engelbrecht, B. S. Lazarov, O. Sigmund, Topology optimization of thermal heat sinks, in: Proceedings of COMSOL conference 2015, 2015.

[26] K. Yaji, T. Yamada, M. Yoshino, T. Matsumoto, K. Izui, S. Nishiwaki, Topology optimization in thermal-fluid flow using the lattice boltzmann method, Journal of Computational Physics 307 (2016) 355 - 377. doi:10.1016/j.jcp.2015.12.008.

[27] L. Laniewski-Wollk, J. Rokicki, Adjoint lattice boltzmann for topology optimization on multi-gpu architecture, Computers \& Mathematics with Applications 71 (3) (2016) 833 - 848. doi:10.1016/j.camwa.2015.12.043.

[28] X. Qian, E. M. Dede, Topology optimization of a coupled thermal-fluid system under a tangential thermal gradient constraint, Structural and Multidisciplinary Optimization 54 (3) (2016) 531-551. doi:10.1007/s00158016-1421-6

[29] J. H. Haertel, G. F. Nellis, A fully developed flow thermofluid model for topology optimization of 3d-printed air-cooled heat exchangers, Applied Thermal Engineering 119 (2017) 10 - 24. doi:10.1016/j.applthermaleng.2017.03.030.

[30] J. Alexandersen, N. Aage, C. S. Andreasen, O. Sigmund, Topology optimisation of natural convection problems, International Journal for Numerical Methods in Fluids 76 (10) (2014) 699-721. doi:10.1002/fld.3954.

[31] J. Alexandersen, O. Sigmund, N. Aage, Large scale three-dimensional topology optimisation of heat sinks cooled by natural convection, International Journal of Heat and Mass Transfer 100 (2016) 876 - 891. doi:10.1016/j.ijheatmasstransfer.2016.05.013.

[32] P. Coffin, K. Maute, A level-set method for steady-state and transient natural convection problems, Structural and Multidisciplinary Optimization (2015) 1-21doi:10.1007/s00158-015-1377-y.

[33] Y. Joo, I. Lee, S. J. Kim, Topology optimization of heat sinks in natural convection considering the effect of shape-dependent heat transfer coefficient, International Journal of Heat and Mass Transfer 109 (2017) 123 133. doi:10.1016/j.ijheatmasstransfer.2017.01.099.

[34] J. Alexandersen, Efficient topology optimisation of multiscale and multiphysics problems, Ph.D. thesis, Technical University of Denmark (2016). 
URL http://orbit.dtu.dk/en/

[35] J. Alexandersen, O. Sigmund, N. Aage, Topology optimisation of passive coolers for light-emitting diode lamps, in: 11th World Congress on Structural and Multidisciplinary Optimization, 2015. doi:10.13140/RG.2.1.3906.5446.

[36] COMSOL Multiphysics v. 5.2a, COMSOL AB, sweden. URL www. comsol.com

[37] B. S. Lazarov, O. Sigmund, K. E. Meyer, J. Alexandersen, Experimental validation of topology-optimised passive coolers, under preparation.

[38] A. Bejan, Convection Heat Transfer, 4th Edition, Wiley, 2013, iSBN: 9780470900376.

[39] A. Bejan, Shape and Structure, from Engineering to Nature, Cambridge University Press, 2000, ISBN: 9780521793889.

[40] F. P. Incropera, D. P. de Witt, Fundamentals of Heat Transfer, John Wiley \& Sons Inc, 1981. 